\title{
SENSOR APPLICATIONS AT KENNEDY SPACE CENTER (KSC)
}

\author{
Jose M. Perotti \\ Acting Lead, Transducers and Data Acquisition Group, \\ National Aeronautics and Space Administration \\ Kennedy Space Center, Florida 32899, USA \\ jose.perotti-1@ksc.nasa.gov
}

\author{
Anthony J. Eckhoff \\ Manager, Transducers and Hazardous Gas Detection \\ DYNACS Incorporated \\ Kennedy Space Center, Florida 32899, USA \\ anthony.eckhoff@ksc.nasa.gov
}

\begin{abstract}
Transducers used at KSC, in support of processing and launch of flight vehicles and payloads, are designed and tested to meet specific program requirements. Any equipment, transducer or support instrumentation in direct contact or in support to flight vehicle operations is considered ground support equipment (GSE) and required to meet strict program requirements (i.e. Space Shuttle Program, Space Station Program, Evolved Expendable Launch Vehicles, etc.)

Transducers used in KSC applications are based on Commercial-Off-The-Shelf transducers and sensor. In order to fully meet $K S C$ requirements, these transducers evolve from standard COTS to modified COTS.

The Transducer and Data Acquisition Group of the Instrumentation Branch at Kennedy Space Center is responsible for providing the technical expertise as well as qualification-testing capability to transform these COTS transducers in modified COTS suitable for use around flight hardware.
\end{abstract}

\section{INTRODUCTION}

Kennedy Space Center (KSC) is the primary launch center for manned rockets in the United States of America. Together with Cape Canaveral Air Force Station (CCAFS), it provides the majority of rocket launches per year in the US and worldwide.

An extensive support infrastructure for the assembly, processing, storage, launch and retrieval of flight vehicles have been in existence since the beginning of the space exploration program.

To support the different phases of assembly, processing, checkout and launch of these vehicles and their payloads, an intricate instrumentation system has been created. There are approximately 3000 different transducers just to support Space Shuttle Pads and Orbiter processing facilities. Of those $3000+$ transducers, approximately eighty percent $(80 \%)$ of them are temperature and pressure transducers.

Transducers used in these facilities are required to meet specific program requirements. Any piece of equipment, transducer or support instrumentation in direct contact or in support to flight vehicle operations is considered ground support equipment (GSE) and required to meet strict program requirements. Depending which program they support (i.e. Space Shuttle Program, Space Station Program, Evolved Expendable Launch Vehicles, etc.) these requirements are slightly different. The instrumentation group at KSC generates and distributes specification documents for each of these GSE transducers, in accordance with program requirements.

Although it is very desirable to maximize the use of commercial-off-the-shelf (COTS) transducers and sensors, in reality these transducers evolve from standard COTS to modified COTS before they meet program requirements.

The Transducer and Data Acquisition Group of the Instrumentation Branch at Kennedy Space Center is responsible for providing transducers and sensors' technical expertise at KSC. The Group also provides qualification-testing capability for transducer certification of all GSE instrumentation utilized by the Space Shuttle Program and the Space Station Program at the Space Station Processing Facility (SSPF). This article will briefly present first some of the program requirements that makes transducers' selection at KSC so specific and unique. Then, the article will provide a few examples of transducers and special tools being utilized in support of vehicle processing and launch applications at KSC.

The authors will also like to recognize that several other efforts in the area of sensors' development and special tools development are currently going on at Kennedy Space Center that are not part of this group and are not included in this article.

\section{SENSOR REQUIREMENTS}

Most of the space vehicles program requirements are formulated based on three main premises. First they are established to assure the safe operation of personnel, flight vehicles and payloads, and support infrastructure. Transducers utilized around the hazardous environments surrounding a flight vehicle must comply with this premise. Secondly, they are established to assure accurate, predictable, repeatable operations to successfully support critical operations. Finally, they are established to assure system and equipment reliability, and low maintainability. The transducers approved for use have to comply with these premises and meet the detailed program requirements.

No less important are requirements designed to assure transducers' interchangeability, commonality, traceability 
and compliance to the existing Launch Processing Systems (LPS).

Transducers qualification testing is performed at $\mathrm{KSC}$ to identify commercial manufacturers and products that meet all these requirements. An average of six months is required to qualify a transducer for GSE applications. This six-month span includes performing all functional and environmental testing as well as working with manufacturers to bring their products to compliance.

Examples of testing designed to verify requirements related to transducers safe operation are normally the following:

- Vibration testing to launch environment levels. Duration of this testing is base normally on 100 launches. It assures not only transducer survivability but also transducer integrity (they do not become damaging debris to flight vehicles or personnel).

- EMI/EMC testing based on MIL-STD-461. This testing assures transducers and equipment does not emit or are susceptible to electromagnetic and radio frequency interference that could affect their nominal operation.

- Materials compatibility testing usually but not exclusively related to hydrogen, oxygen and hypergols $\left(\mathrm{MMH}, \mathrm{N}_{2} \mathrm{O}_{4}\right)$.

Testing is also performed to assure accurate, predictable, repeatable measurements are provided to support critical operations. As example, the following testing and analytical calculations are performed:

- A series of at least three calibration runs with at least 6 calibration points $(0,20,40,60,80$, and $100 \%$ of Full Scale) on each run, both upward and downward directions.

- Linearity, repeatability, hysteresis, accuracy and error band calculations to assure measurement precision.

To assure measurement and transducers reliability, long term testing and accelerated aging testing is performed. Among them:

- Temperature testing in the industrial temperature range of -25 to $85^{\circ} \mathrm{C}$. Calibration runs are performed at each temperature steps and calculations related to accuracy and error band are performed.

- Drift (8 hours) and Stability (90 days) testing is performed to assure longevity of transducers.

Additionally, testing is also performed to assure compatibility to the Launch Processing System (LPS), among others.

Again, the origin of these transducers is COTS products, normally from the aerospace division of a commercial manufacturer. By the end of the process, the result is a modified version of these COTS to meet program requirements.

\section{SENSORS APPLICATIONS}

\section{A) CRYOGENIC APPLICATIONS}

Temperature sensors are the most commonly used transducers in cryogenic applications at KSC. Traditionally, they are platinum resistance temperature devices (RTDs) sensors, with high purity platinum material $(\alpha=0.000395)$ and $R_{0}$ of $1,400 \Omega$. They are used where high accuracy, high signal-to-noise ratio and narrow temperature ranges are required. Thermocouples (TC) temperature probes are used where a less stringent application is performed. These PRT probes are COTS temperature sensors, enclosed in a specially designed and tested stainless steel housing to withstand vibration and bending stresses from the environment they are in (Figure 1). Advancements in silicon diode technology, coupled with the lack of availability of these 1,400 $\Omega$ probes, prompted the Transducers Group to fully qualify silicon diode temperature probes for cryogenic applications. This technology works great at liquid hydrogen (LH2) temperatures. It provides excellent sensitivity, signal-to-noise ratio, and accuracy. Unfortunately, they under-perform PRTs at liquid oxygen temperature ranges. The Transducers Group is presently qualifying a high purity platinum temperature probe $(\alpha=0.0003916)$ with $R_{0}$ of $1,000 \Omega$.

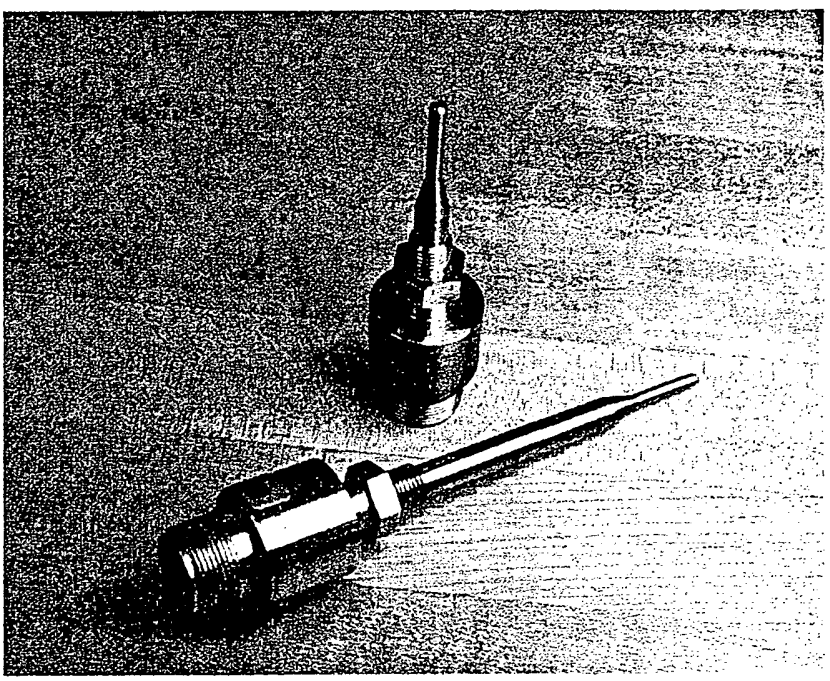

Figure 1. - Cryogenic Temperature Probes

Cryogenic flow measurements are also a critical application at KSC. Flow meters are used to quantify the loading of liquid oxygen in the External Tank (ET) of the Space Shuttle. These meters have to work at liquid oxygen temperatures and be accurate to below $1 \%$ of full scale. There are not many technologies that meet these requirements. Presently, the Transducers Group is working to qualify 2 inch diameter, Vortex Shedding-type flow meters to support these needs (Figure 2). 


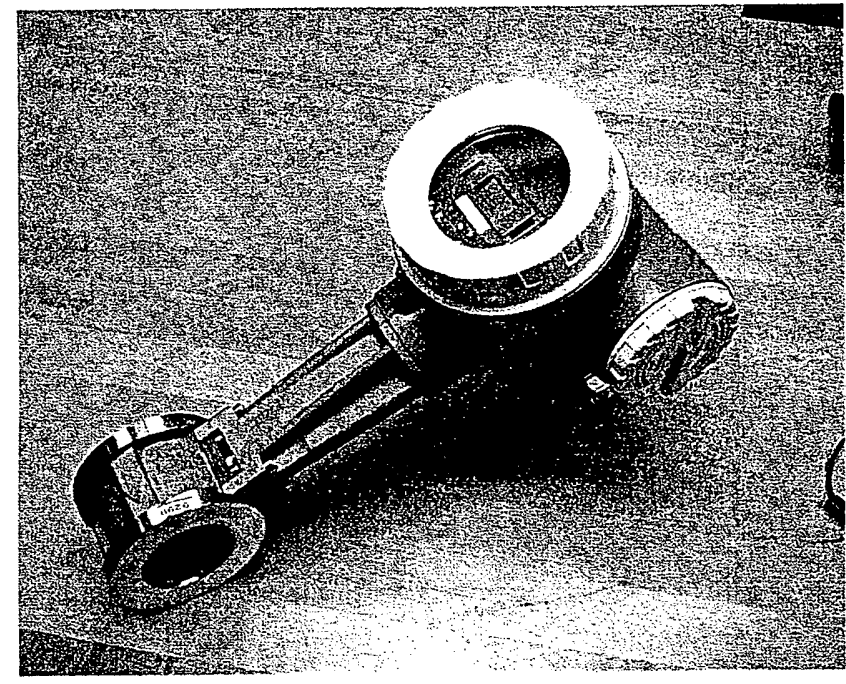

Figure 2. - Vortex Shedding Flowmeter Used in Cryogenic Applications

\section{B) HYPERGOL APPLICATIONS.}

Another application where COTS transducers and test equipment are used to support Shuttle Operations is the flow calculation of Hypergol (MMH and N2O4) commodities. It is required to accurately know the amount of commodities loaded in the Space Shuttle Orbiter every mission. These commodities are used by the orbital maneuvering systems to steer the Orbiter in space. Flow meters used previously at the launch pads were turbine flow meters and historically have had the problem of responding to not only liquid flowing but also to bubbles in the system, thus rendering inaccurate measurements. Furthermore, bearing damage, due to over spinning as a resulting from gas in the lines, was prevalent. Additionally, they trapped toxic hypergol commodities inside, thus creating a potential hazardous condition for personnel calibrating and replacing these units.

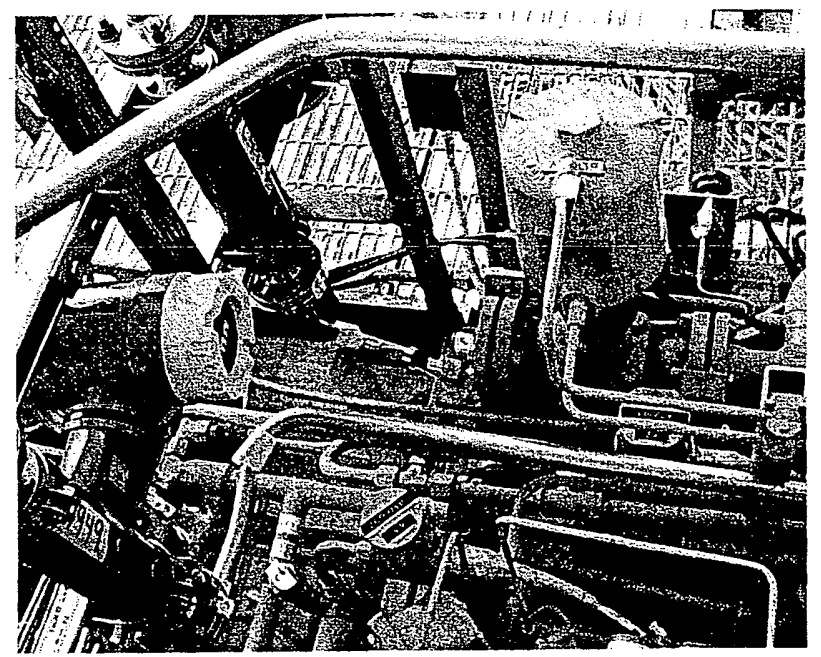

Figure 3. - Coriolis Flowmeter Used in Hypergol Applications
A new straight tube Coriolis-type flow meter has been qualified for used at the launch pads for hypergol applications. The new meter is a mass flowmeter, which accounts for bi-phase flow and reverse flow accurately. The meter is very accurate $(0.2 \%$ of $F S)$, although $\mathrm{KSC}$ has qualified the meter under Shuttle Operations conditions as a $1 \%$ device (recognizing that some of the uncertainties come from the test equipment itself). Several modifications were made to the meter to comply with KSC requirements, mainly in the areas of connectors and seals (to be compatible with hypergol media). The meters are presently installed in launch Pad A and have supported several hypergol flows successfully (Figure 3 ).

\section{C) SPECIAL TOOLS}

Tire pressure monitor (TPM) Shuttle Operations requirements call for performing pressurization and pressurization decay tests to validate the Shuttle Orbiter tires and struts for flight, to identify any leak paths during processing before the Orbiter is ready for flight. To achieve this task, an instrumentation system capable of working at 450 pounds per square inch absolute (PSIA) and detecting 0.1-psi changes is required. The request and design of the new tool was prompted by safety concerns related to the long pressurized lines of the existing system, as well as borderline accuracy vs. needs of the existing Shuttle instrumentation. The Tire and Strut Pressure Monitor (TPM) project goal was to design a handheld system to accurately measure Orbiter Tire and Strut pressure and temperature, thus removing the need for the large and unwieldy system presently in use.

The project team performed an extensive market research to select small, highly accurate pressure and temperature sensors to incorporate in the design. Based on these sensors, the team started the design of the optimum mechanical interface between the tire/strut valve and the sensors, and the design of a compact, hand-held electronics unit that provided rapid, reliable pressure/ temperature data display. COTS pressure and temperature sensors were selected and a housing was designed for attachment as close to the tire or strut measurement location as possible, allowing the user to make accurate measurements rapidly. This minimized the length of high-pressure lines and allowed reasonable distance from the tire or strut to the operator.

The pressure measurement portion of the proposed system centers on a small, highly accurate pressure sensor. The commercially available sensor is capable of delivering pressure measurements repeatable to within 0.03 PSIA. Temperature compensation and correction is required to maintain this tight tolerance through out the wide operating temperature range. The complete pressure sensor assembly, housed in a stainless steel enclosure, contains the pressure sensor, a temperature sensor and electronics for precision excitation to the pressure sensor and amplification of pressure and temperature measurements. The sensing unit 
housing, fittings and unit's orientation was designed to allow for easy installation and removal by technicians.

The Tire and Strut Pressure Monitor user interface is a hand-held device that can be powered by 12 -VAC or by 9 VDC batteries. Within the user interface, electronics provide supply voltage to the sensing unit electronics, as well as low pass filtering and analog-to-digital conversion of sensors measurements. The system includes a smart software algorithm embedded in a micro-controller. Utilizing complex conversion equations developed from pressure and temperature sensor calibration data; the system is capable to optimize measurements accuracy at any given operating temperature. Thanks to the developed software algorithm, the system is capable of maintaining the required accuracy throughout the required temperature range (20 degrees to 120 degrees Fahrenheit). The hand-held electronics provide the user with an easily read visual display of pressure / temperature or the streaming of pressure / temperature data via an RS-232 interface (Figure 4).

Additionally, calibration software will allow for calibration data to be automatically utilized for the generation of new data conversion equations, simplifying the calibration processes that are so critical to reliable measurements.

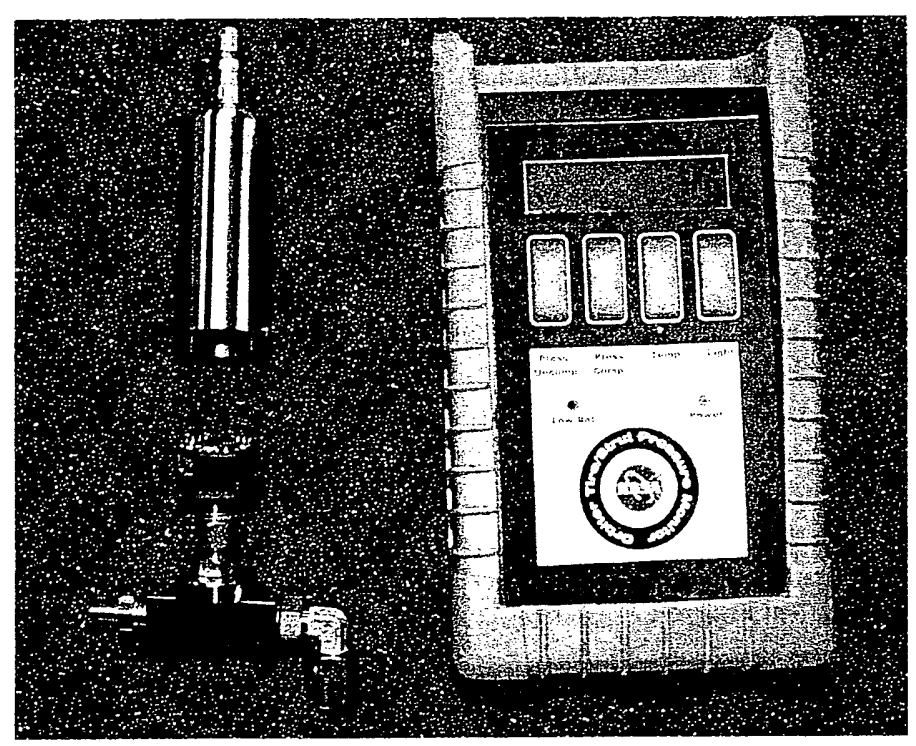

Figure 4. - Tire Pressure Monitor (TPM) sensor head and handheld unit.

\section{ET Centering and Alignment System (ET CAS): When}

the External Tank (ET) is mated to the Solid Rocket Boosters (SRB); it is necessary that the ET be precisely positioned. The ET CAS is a tool that assists personnel in this operation by providing an automated measurement of the distance that separates the ET from each SRB. The ET CAS also provides a laser-generated line that assists personnel in aligning the ET such that the two SRBs and the ET have the same centerline. The current ET CAS uses two sensor units that each contain an acoustic sensor and a laser crosshair. These are connected to a central display unit by long cables that provide power to the sensors and a path for the measurement data. The sensor units are attached to the SRB by magnets. These acoustic sensors require frequent calibration and are suspect at temperature extremes.

The enhanced system being developed is a wireless, laserbased centering tool. It uses a new COTS laser distance sensor to measure the distance between the SRB and the ET and also provide a line for the centerline adjustment. Laboratory testing is characterizing the sensor's performance over the required temperature range. A temperature measurement will be used in the system to compensate for environmental changes.

The new system uses wireless technology to communicate to a new User Interface. The core of the communications system is based on a wireless system that was also developed in the Transducer and Data Acquisition Group. This wireless system will be integrated with the laser distance sensor, a custom control and power circuit, and a battery pack to create the sensor unit (Figure 5). This new sensor unit package will be magnetically attached to the SRB to support the operations. The new User Interface has a small color LCD touch screen panel that will show the relative position of the ET to the SRB both numerically and graphically.

The new ET CAS will have a number of advantages over the current system. The laser distance sensor will provide greater accuracy and will not require calibration. The entire system will fit in a case the size of a deep briefcase. The

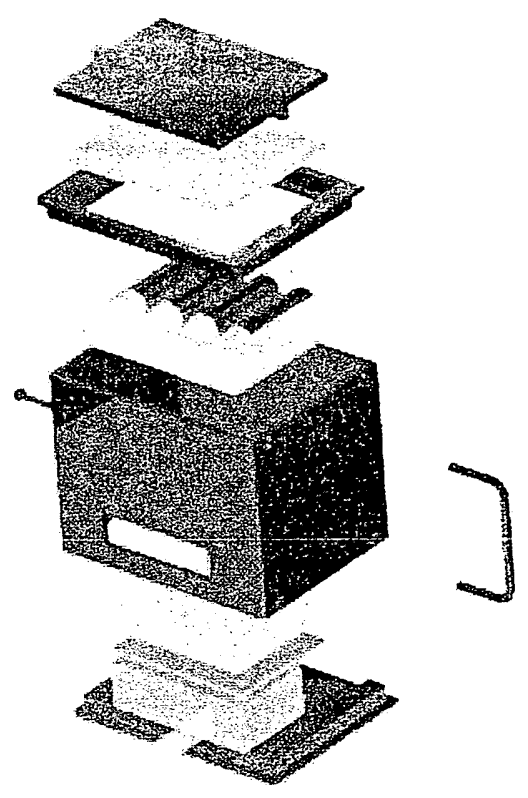

wireless system will allow the engineer, using the interface display unit, to relocate out of the way of the operation. 


\section{SUMMARY}

Transducers used at KSC in support of processing and launch of flight vehicles and payloads are required to meet specific program requirements. Any equipment, transducer or support instrumentation in direct contact or in support to flight vehicle operations is considered ground support equipment (GSE) and required to meet strict program requirements (i.e. Space Shuttle Program, Space Station Program, Evolved Expendable Launch Vehicles, etc.)

Although it is very desirable to maximize the use of commercial-off-the-shelf (COTS) transducers and sensors, in reality, these transducers evolve from standard COTS to modified COTS before they meet program requirements.

The Transducer and Data Acquisition Group of the Instrumentation Branch at Kennedy Space Center is responsible for providing transducers and sensors' technical expertise, as well as providing qualification testing capability for transducer certification of all GSE instrumentation utilized by the Space Shuttle Program and the Space Station Program at the Space Station Processing Facility (SSPF). 
E. 